Revista de Biología Marina y Oceanografía

Vol. 48, №3: 653-660, diciembre 2013

DOI 10.4067/S0718-19572013000300023

Nota Científica

\title{
Inducción al desove de la navaja Ensis macha mediante inyección de serotonina
}

Induction of spawning of the razor clam Ensis macha by injection of serotonin

\author{
Daniel Arriagada', Irene Lépez ${ }^{1}$, Maryori Ruiz ${ }^{2}$ e Ingrid Contreras ${ }^{1}$ \\ ${ }^{1}$ Laboratorio Ingeniería Acuícola Irene Lépez Ltda., Gobernador Juan Henríquez 910, Concepción, Chile. \\ danielarriagadaobregon@gmail.com \\ ${ }^{2}$ Programa de Doctorado Sistemas Marinos Costeros, Facultad de Recursos del Mar, Universidad de Antofagasta, Casilla 170, \\ Antofagasta, Chile
}

\begin{abstract}
The effect of serotonin spawning stimulating agent, was evaluated in Ensis macha sexually mature broodstock, conditioned in laboratory. Neurotransmitter $0.9 \mathrm{ml}$ at a concentration of $10^{-5} \mathrm{M}$ was injected into the foot of each specimen. 5 -HT induced gamete release in $92 \%$ of injected broodstock, always producing the fastest response in males, removing sperm at $36 \mathrm{~min}( \pm 15.9)$ after injection. The release of mature oocytes began at $170 \mathrm{~min}( \pm 42.4)$ post-injection. Embryonic development to the veliger larva formation, straight hinge $D$ had a survival of $88 \%$, no malformations have been observed in larvae. In only one trial, fertilized oocytes were not viable. Finally, we can confirm that there is a pattern in the release of mature gametes E. macha broodstocks due to the action of serotonin 5-HT and could be a useful tool for aquaculture practices.
\end{abstract}

Key words: Ensis macha, serotonin, spawning

\section{INTRODUCCIÓN}

En Chile se destaca el bivalvo enterrador Ensis macha (Molina, 1782), especie conocida comúnmente como 'navaja de mar', 'huepo' o 'machuelo', cuya distribución geográfica incluye el Pacífico desde Caldera (27³'S; $70^{\circ} 52^{\prime} \mathrm{O}$ ) hasta la región Magallánica (56³0’ S; 7054’O) y en el Atlántico Sur (Ramírez 1993). Se encuentra asociado a fondos blandos en sedimentos de arena gruesa, principalmente en el submareal de playas arenosas del centro y sur de Chile, formando agregaciones desde los 4 a $10 \mathrm{~m}$ de profundidad. E. macha desova en los meses de primavera-verano con 2 pulsos de desove en Chile y Argentina (Urban 1996, Avellanal et al. 2002, Aracena et al. 2003, Barón et al. 2004).

Este bivalvo enterrador constituye un importante recurso para la pesca artesanal de la zona sur de Chile, especialmente en las regiones del Biobío, Los Ríos, Los Lagos y Magallanes, con registros de desembarques de 4.345 ton durante el 2011 (SERNAPESCA 2011) y debido a la alta demanda por parte de la industria procesadora, los bancos naturales han sido explotados en forma importante. En respuesta a la alta presión de pesca en esta especie (Lépez et al. 2004¹), el cultivo de E. macha se investiga desde 1990 y a pesar de los esfuerzos y tiempo de investigación, al presente no existe tecnología que permita industrializar la producción de este organismo.

La mayoría de los bivalvos marinos son especies ovíparas cuyos gametos son liberados al ambiente donde acontece la fecundación; proceso que puede ser desencadenado por inductores mecánicos, físicos y ambientales (Bayne 1976, Ferran 1993, Guerra \& Villagarcía 1993). Varios métodos de inducción de desove artificial han sido descritos con la finalidad de producir estadíos tempranos en las plantas productoras de semillas, siendo el estrés térmico y/o adición de espermatozoides y ovocitos los más utilizados (Helm et al. 2006). La inducción artificial de desove en los centros de acuicultura no siempre asegura la liberación de gametos por parte de los reproductores, lo que podría ser atribuido a que el proceso de gametogénesis no se ha completado aún o no es coincidente con su comportamiento en el medio natural, como ocurre en Ensis arcuatus (Martínez \& Da Costa 2008). Martínez (2008) señala que el proceso de gametogénesis se detiene con los ovocitos en profase I de la meiosis y se reinicia posteriormente, cuando la

${ }^{1}$ Lépez MI, D Arriagada \& C Padget. 2004. Protocolo cultivo de la navaja Ensis macha. Proyecto PPT D01T2021. Universidad de Concepción, Concepción, 18 pp. (Registro PINo 140569). 
vesícula germinativa se rompe, dependiendo de la especie, antes o después de que los gametos se liberan al medio. En Argopecten purpuratus, Martínez et al. (2000b) demostraron que el proceso de ruptura de la vesícula sucede antes de que sean liberados al ambiente y Leclerc et al. (2000), señalan que en los bivalvos Ruditapes y Crassostrea la vesícula germinal se rompe después de que son liberados al medio.

En moluscos marinos el reinicio de la meiosis obedece a un proceso natural de maduración del ovocito, y es desencadenado por señales extracelulares o bien en algunos casos, por contacto con espermatozoides. También el reinicio de la meiosis puede ser inducido por la aplicación de sustancias que inciden en el metabolismo endocrino de los moluscos. Entre las vías del metabolismo endocrino de vertebrados e invertebrados (Helm et al. 2006) más estudiadas para inducir el desove, está el 5-HT (5-hidroxitriptamina) un componente de la serotonina, perteneciente al grupo de las monoaminas biogénicas (i.e., dopamina, noradrenalina e histamina) y ampliamente reconocido por su efecto neurohormonal en procesos reproductivos en varias especies de moluscos bivalvos. Se ha indicado que induce la liberación de gametos en trozos de gónada (Wang \& Croll 2003), estimula el desove (Ram et al. 1996), produce reactivación de espermatozoides (Kadam \& Koide 1990) y maduración de ovocitos (Gobet et al. 1994 y Deguchi \& Osanai 1995). Experimentos in vitro señalan que 5-HT induce la ruptura de la vesícula germinal en algunos bivalvos y que participa en el proceso de metamorfosis y asentamiento larval en el gastrópodo Haliotis asinina (Wang et al. 2010). La serotonina está presente en las gónadas de bivalvos, tanto en el epitelio germinal como en los folículos, en el gonoducto y en larvas de mitílidos (Paulet et al. 1993, Pani \& Croll 1995, Croll et al. 1995, Flyachinskaya 2000, Garnerot et al. 2006). Asimismo, en el bivalvo Argopecten purpuratus las hormonas serotonina y prostaglandinas $\mathrm{E}_{2}$ y $\mathrm{F}_{2 \alpha}$ han demostrado incrementar el porcentaje de ovocito en estado de ruptura de vesícula germinal (Martínez et al. $2000 \mathrm{~b}$ ), lo que permite que estos alcancen la metafase I y por consiguiente la fecundación. Wang (2000), en el ostión Patinopecten yessoensis demostró que la aplicación de serotonina a una concentración de $10^{-5} \mathrm{M}$ incrementó significativamente el número de huevos liberados, los cuales tuvieron un desarrollo normal transcurrida la fertilización. Gibbons \& Castagna (1984) obtuvieron desoves exitosos a una concentración $2 \mathrm{mM}$ de serotonina en 6 especies de moluscos bivalvos: en Argopecten irradians, Crassostrea virginica y Spisula solidissima al ser inyectados en el tejido intragonadal y en Arctica islandica, Geukensia demissa y Mercenaria mercenaria mediante inyecciones en el músculo aductor anterior. Wang \& Croll (2003) demostraron que en Placopecten magellanicus la combinación de estradiol $10^{-6} \mathrm{M}$ con serotonina $10^{-5} \mathrm{M}$ potencia la liberación de gametos. Respecto a esto, se ha señalado que el estradiol $\left(\mathrm{E}_{2}\right)$ induce la formación de receptores de 5-HT (Cubero et al. 2010, Sánchez et al. 2011).

La presencia y función de la serotonina de estimular el desove en algunos representantes de la clase bivalvia permite plantear la hipótesis que este agente neurohormonal podría tener un efecto similar en el solénido E. macha. Al presente, no existen investigaciones que determinen el efecto de la serotonina en el desove de esta especie, conocimiento que podría constituir una útil herramienta para inducir la liberación de gametos permitiendo controlar la fecundación y optimizar la supervivencia de larvas ' $D$ ' para fines de cultivo y estudios ecotoxicológicos. En consecuencia, el objetivo de la presente investigación fue testear el uso de la serotonina como agente estimulador de desove en ejemplares madurados en laboratorio, del bivalvo Ensis macha, y la viabilidad de los gametos obtenidos mediante esta técnica (eclosión de huevos y larvas veliger fase D) a través de inyecciones de serotonina (5-hidroxitriptamina, complejo sulfato creatinina) en las estructuras gónadapie.

\section{MATERIALES Y MÉTODOS}

\section{OBTENCIÓN DE LOS REPRODUCTORES Y DISEÑO EXPERIMENTAL}

Se extrajeron ejemplares adultos de Ensis macha, mediante buceo, desde bancos naturales de la Región del Biobío en septiembre y octubre y fueron llevados al laboratorio de la Empresa Ingeniería Acuícola Irene Lépez Ltda, ubicada en la Región del Biobío, Chile (36 50'S; 7305'O) para su acondicionamiento gonadal. Los reproductores fueron acondicionados en 5 contenedores de $50 \mathrm{~L}$ con fondo arenoso durante 45 días en un sistema de recirculación, con aireación constante, agua de mar filtrada $(0,5 \mu \mathrm{m})$, temperada a $17^{\circ} \mathrm{C}( \pm 1)$ y a una salinidad de $32( \pm 1)$. Su alimentación diaria estuvo basada en una mezcla de microalgas cultivadas en el mismo laboratorio. Estas fueron: Isochrysis galbana (clon T-iso), Phaeodactylum tricornutum, Tetraselmys tetrathele y Chaetoceros muelleri (González et al. 1995), en una relación 1:1:1:1 y a una concentración final, medida a través de una cámara 
Tabla 1. Longitud valvar de reproductores de E. macha inyectados con serotonina. Cada valor representa la media \pm la desviación estándar (DS) / Valvar length of broodstocks $E$. macha injected with serotonin. Each value represents the mean \pm standard deviation (SD)

\begin{tabular}{lcccc}
\hline Ensayo de inducción & $\begin{array}{c}\text { Longitud valvar } \\
(\mathrm{cm})\end{array}$ & $\begin{array}{c}\text { Inyectados } \\
\mathrm{N}\end{array}$ & $\begin{array}{c}\text { Reproductores } \\
\text { desovados } \\
(\%)\end{array}$ & $\begin{array}{c}\text { Ovocitos desovados } \\
\text { por hembra } \\
\left(\mathrm{N}^{\circ}\right)\end{array}$ \\
\hline Ensayo 1 (9 nov) & $13,84(0,7)$ & 7 & 100 & $855.000(56.423)$ \\
Ensayo 2 (15 nov) & $13,34(0,7)$ & 7 & 100 & $820.000(908.717)$ \\
Ensayo 3 (21 nov) & $13,44(1,0)$ & 6 & 100 & $1.470 .200(966.979)$ \\
Ensayo 4 (4 dic) & $11,75(1,0)$ & 6 & 66,7 & $426.000(359.203)$ \\
Ensayo 5 (11 dic) & $15,17(1,0)$ & 10 & 90 & $1.898 .880(2.093 .985)$ \\
\hline
\end{tabular}

de Neubauer, que fluctuó en un rango de 100.000 a 150.000 cél $\mathrm{ml}^{-1}$ (Lépez et al. 2011). Un total de 36 reproductores maduros de E. macha de 13,5 $( \pm 1,2) \mathrm{cm}$ de longitud valvar y de 45,3 $( \pm 14,8) \mathrm{g}$ de peso húmedo (Tabla 1$)$ se obtuvieron del sistema de acondicionamiento, en noviembre y diciembre 2012.

Previo a cada ensayo, se realizó una observación macro y microscópica mediante un frotis de tejido gonadal, de reproductores de navaja acondicionados, para evaluar el grado de desarrollo gonadal y determinar el estado de madurez según el índice macroscópico de madurez (MMS) descrito por Aracena et al. (2003), relacionándolo con la escala gametogénica desarrollada para el solénido Ensis arcuatus por Darriba et al. (2004), que reconoce 7 estados de condición gametogénica: E0: reposo sexual, EI: inicio gematogénesis, EII: gametogénesis avanzada, EIIIA: madurez, EIIIB: inicio de puesta, EIIIC: restauración y EIV: agotamiento. El MMS describe 3 estados de madurez: Estado 1, el ovario cubre hasta el $50 \%$ de la glándula digestiva y su volumen y granulación es mínima o intermedia: Estado 2, el ovario cubre la mitad de la glándula digestiva y su volumen es intermedio y la granulación está al máximo: Estado 3: la glándula digestiva está completamente cubierta por el ovario, el volumen y granulación están al máximo.

Para la inducción a desove con serotonina se realizaron aproximadamente 5 muestreos sistemáticos (ensayos) cada 7 días, extrayendo 6 a 10 reproductores acondicionados de E. macha por muestreo (Tabla 1). Los reproductores se depositaron en una bandeja plástica de $40 \mathrm{~L}$, con agua de mar temperada a $17^{\circ} \mathrm{C}$, filtrada a $0,5 \mu \mathrm{m}$ y esterilizada con UV. Previo a la inducción, la serotonina cristalina (5hydroxytryptamine creatinine sulfate complex, SigmaAldrich) fue solubilizada en $\mathrm{HCl} 0,1 \mathrm{M}$ en agua destilada, y neutralizada con $\mathrm{NaOH}$ 0,1M. Subsiguientemente, un volumen de $0,9 \mathrm{ml} 10^{-5} \mathrm{M}$ de serotonina se inyectó en la región postero-ventral de cada individuo, específicamente, en el complejo gónada-pié (Darriba \& López 2011), y posteriormente fueron devueltos a la bandeja con agua de mar. La concentración empleada fue la óptima $\left(10^{-5} \mathrm{M}\right)$ utilizada por Wang (2000), en la inducción a desove mediante serotonina en el ostión Patinopecten yessoensis.

Posteriormente, los machos inyectados se separaron de las hembras mediante la observación de gametos masculinos liberados (siendo estos los primeros en desovar), y se depositaron en forma conjunta en un balde plástico de 5 L con agua de mar, para recolectar solución de espermatozoides. En tanto las hembras fueron dispuestas en forma independiente en matraces de $2 \mathrm{~L}$. El desove se produce después de $2 \mathrm{~h}$ de ser inyectadas, momento en el cual se realiza la fecundación in vitro mediante la incorporación de $15 \mathrm{ml}$ de solución de espermatozoides a cada matraz en una relación de 1 ovocito : 100 espermatozoides (Lépez et al. 2004¹, 2011). En los animales inyectados, se determinó el tiempo de respuesta de inicio y duración del desove.

\section{EFecto de SERotonina EN LA LIBERACión de gametos (ECLOSIÓN DE HUEVOS Y LARVAS D)}

Para determinar el efecto de la serotonina en el proceso de desove, previo a la fecundación in vitro, se obtuvo una muestra de $0,5 \mathrm{ml}$ de cada matraz y se contó el número de ovocitos liberados por hembra en una cámara de contaje, constituida por una placa petri con fondo cuadriculado, mediante un microscopio óptico, modelo Olympus ${ }^{\circledR}$ CX21. Para evaluar el efecto de la serotonina en la viabilidad de los gametos, se utilizó la eclosión de 
los huevos (fecundados) y larvas veliger fase D como indicadores. En el primero de cada matraz, se obtuvo una muestra de $0,5 \mathrm{ml}$ y se visualizó en un microscopio óptico el número de ovocitos fecundados, utilizando los criterios de fecundación señalados por Lépez et al. (2011): presencia de la membrana de fecundación y liberación del primer y segundo corpúsculo polar por finalización de meiosis I y II, respectivamente. Para determinar el segundo indicador, a las 48 h post-fecundación se obtuvo una muestra de $0,5 \mathrm{ml}$ de cada matraz y se determinó el número de larvas veliger fase $\mathrm{D}$.

\section{Análisis estadísticos}

En todos los análisis estadísticos la hipótesis nula se rechazó con un valor de $\alpha \leq 0,05$. Para cada variable muestreada se constató la normalidad de los datos con el test Kolmogorov-Smirnov y la homogeneidad de varianza. Se aplicó la transformación de logaritmo base 10 a la variable de fecundidad. Se aplicó un test de Kruskal Wallis al tiempo inicio de desove, considerando el ensayo con serotonina como factor fijo debido a que los datos no tenían una distribución normal y las varianzas no eran homogéneas. Se utilizó ANDEVA de una vía para determinar el efecto de la serotonina en la fecundidad de las hembras y tiempo de duración de desove de éstas, considerando el ensayo con serotonina como factor fijo (Quinn \& Keough 2002). Todos los análisis se realizaron en el programa Minitab 15 (Minitab Inc.).

\section{RESULTADOS Y DISCUSIÓN}

\section{MADUREZ DE REPRODUCTORES ACONDICIONADOS}

Se caracterizaron microscópicamente muestras de tejido gonadal de reproductores acondicionados por presentar un volumen significativo del tejido gonadal sobre el músculo aductor anterior, cubriendo por completo la glándula digestiva e invadiendo el interior del pie. También se observó tejido gonadal granular en las hembras y lechoso en los machos. Observaciones al microscopio de frotis de tejido gonadal mostraron en machos, espermatozoides muy activos al contacto con el agua de mar y en hembras, ovocitos maduros libres, con bordes redondeados o con forma poliédrica, con cromatina difusa y citoplasma de gran tamaño. Con estas características se determinó que los reproductores de $E$. macha acondicionados se encontraban en una fase EIIIA (madurez) del ciclo gametogénico al inicio de cada ensayo y con un índice macroscópico de madurez $\mathrm{MMS}=3$.

\section{EFecto de la SERotonina EN LA LIBERACión DE GAMETOS}

Se ha señalado que inyecciones de serotonina (5-HT) estimulan el desove en una amplia variedad de bivalvos (Matsutani \& Nomura 1982, Gibbons \& Castagna 1984, Wang 2000, Wang \& Croll 2003), observándose la misma respuesta en este estudio con reproductores de E. macha. De los 36 reproductores de E. macha sometidos a inducción a desove, el $92 \%$ respondieron positivamente a las inyecciones con serotonina $10^{-5} \mathrm{M}$. Los machos liberaron espermatozoides activos que fueron capaces de fecundar más de 20 millones de ovocitos obtenidos en los 5 ensayos (Tabla 1). Las 17 hembras liberaron, en promedio, 1.151.847 ovocitos, siendo las hembras de menor tamaño las que liberaron una menor cantidad de ovocitos, 426.000 (ensayo 4, Tabla 1) y las de mayor tamaño evacuaron 1.898 .880 ovocitos, presentando un coeficiente de correlación (R2) entre el tamaño del individuo (x) y número de ovocitos (y) de 0,92 ( $\left.\mathrm{y}=2943 * e^{0,424 x}\right)$.

La respuesta obtenida en Ensis macha fue similar a lo obtenido en inducciones realizadas en otros moluscos con serotonina, donde actuaría como una neurohormona estimulando varios procesos a nivel celular vía mecanismos de fosforilación de las proteínas quinasas (Campos et al. 1992). Este proceso desencadenaría contracciones del sistema reproductor femenino y masculino de E. macha, que permitirían la liberación de gametos, sin alterar la competencia de los ovocitos y/o procesos posteriores de fecundación, desarrollo embrionario y larval temprano siempre que los reproductores estén en un estado de madurez apropiado.

La concentración de 5-HT, empleada en este estudio $\left(10^{-5} \mathrm{M}\right)$, fue la concentración óptima utilizada por Wang \& Croll (2003) en el ostión Patinopecten yessoensis. Matsutami \& Nomura (1987) señalan que concentraciones de serotonina superiores a $10^{-6} \mathrm{M}$, generan un rápido decrecimiento en el porcentaje de liberación de gametos en la misma especie. Esto explica que el $92 \%$ de los reproductores de Ensis macha, utilizados en este estudio, respondieron positivamente a la inducción con serotonina.

\section{TIEMPO DE RESPUESTA DE REPRODUCTORES INDUCIDOS CON SEROTONINA}

En los 5 ensayos, los reproductores machos de E. macha fueron los primeros en empezar a liberar sus gametos (Fig. 1 ), con un tiempo promedio de respuesta de $36 \mathrm{~min}( \pm$ $15,9)$ post-inyección con serotonina, siendo la respuesta 


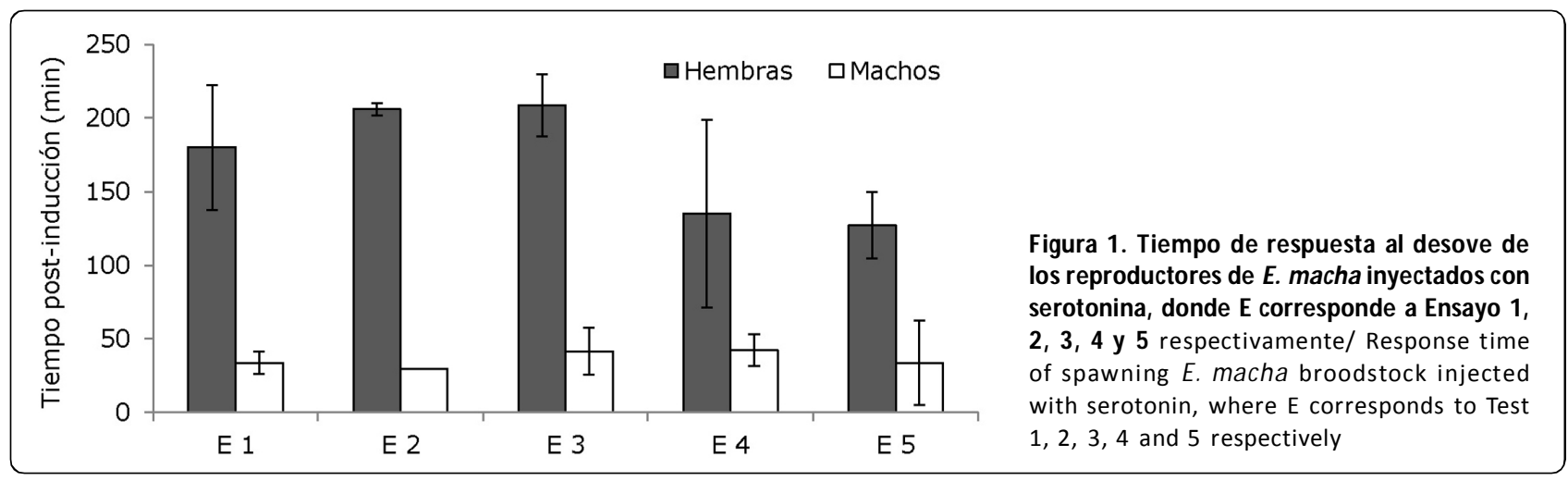

más rápida a los 10 min (ensayo 5). Las hembras iniciaron el proceso de desove en un tiempo significativamente más tardío (Kruskal Wallis, $P<0,05$ ), con un promedio de 170 min $( \pm 42,39)$ post-inyección, siendo la respuesta más rápida a los 90 min, observada en los ensayos 4 y 5 (Fig. 1). Este tiempo de inicio de desove en los 5 ensayos, fue mayor a lo referido por Gibbons \& Castagna (1984) en los bivalvos Argopecten irradians, Crassostrea virginia, Spisula solidisima, Arctica islandica, Geukensia demissa y Mercenaria mercenaria, cuyos tiempos fueron menores a $30 \mathrm{~min}$, desconociéndose si los machos empezaron a evacuar primero que las hembras como se describe en este artículo. En 6 especies de la almeja del género Tridacna, el efecto de la serotonina inyectada fue muy rápido obteniéndose la liberación de espermatozoides entre 1,7 y 3,8 min (Braley 1985). En este estudio, los primeros en ser liberados fueron los espermatozoides. En algunas especies, como el ostión Amusium pleuronectes, sólo hubo liberación de espermatozoides al ser inducidos con serotonina, debiendo realizar un 'stripping' de la gónada femenina para fertilizar ovocitos (Belda \& Del Norte 1988).

\section{TIEMPo de DURACión DEL DESOVE}

El tiempo durante el cual desovaron las hembras de $E$. macha presentó una respuesta similar en los 5 ensayos, sin diferencias significativas entre ellos (ANDEVA $F_{1,57}$, $P>0,05)$. El tiempo de duración promedio fue de $43 \mathrm{~min}$ $( \pm 21,44)$;el menor tiempo de desove fue de $15 \mathrm{~min}, \mathrm{y}$ observado en el ensayo 2, mientras que el más prolongado duró 105 min y se produjo en el ensayo 5 (Fig. 2). En los 5 ensayos realizados con serotonina, se observó una respuesta similar en la cantidad de ovocitos liberados (ANDEVA $\mathrm{F}_{0,87} P>0,05$ ), que tiene relación directa con el tiempo de desove, observándose que la hembra del ensayo 5 que más tiempo estuvo desovando (105 min), liberó la mayor cantidad de gametos, 5,7 millones $( \pm 4,60)$ de

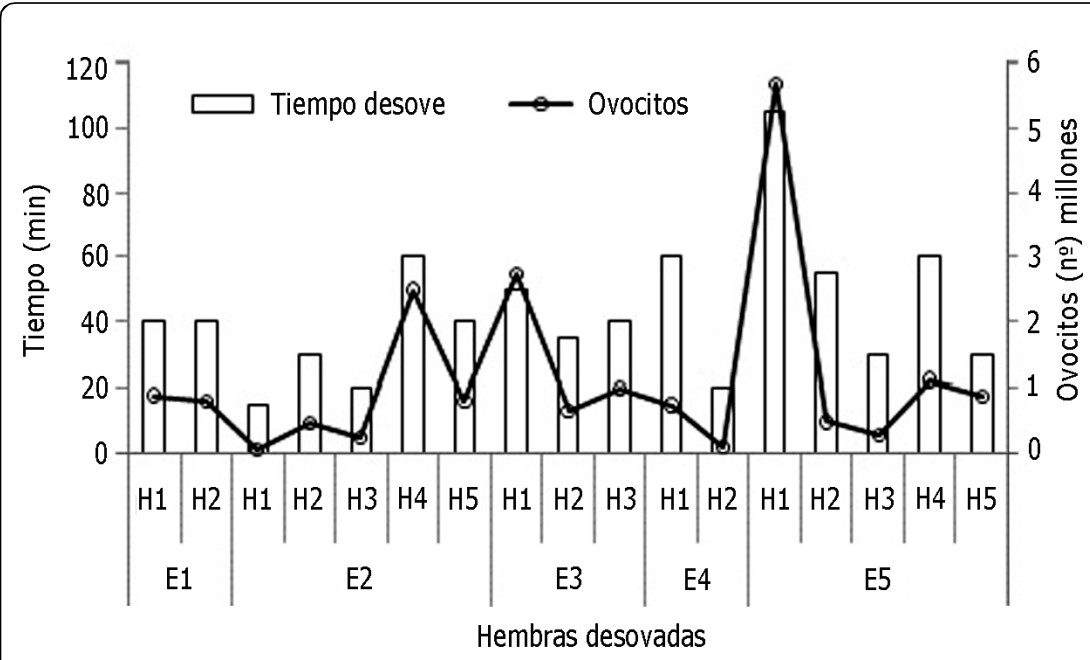

Figura 2. Duración de desove y cantidad de ovocitos desovados por E. macha inducidas con serotonina. $E$ corresponde a ensayos 1, 2, 3, 4 y 5 y H corresponde a hembras / Duration of spawning and oocytes number released by $E$. macha induced with serotonin. E corresponds to tests $1,2,3,4$ and 5 and $\mathrm{H}$ corresponds to females 
ovocitos, mientras que las hembras que menor tiempo estuvo desovando, $15 \mathrm{~min}$ (ensayo 2), liberó la menor cantidad promedio de gametos, $72.000( \pm 12,18)$ ovocitos (Fig. 2). Estos resultados tienen una relación directa con el tamaño del individuo, como se ha mencionado anteriormente.

Aunque no hubo diferencias significativas en el número de ovocitos liberados por el rango de talla utilizado en este trabajo, sí se observó un coeficiente de correlación alto entre la talla de las hembras y el número de ovocitos y que también se expresa en el tiempo de duración del desove. Las altas desviaciones estándar explican el porque las diferencias entre ellas no resultaron significativas. La alta correlación entre la talla de las hembras y el número de ovocitos liberados descarta un probable efecto del grado de madurez de las hembras.

\section{CONTROL DE VIABILIDAD DE OVOCITOS FECUNDADOS}

El porcentaje de fecundación de los ovocitos desovados fluctuó entre el 80 y el 99\%. El valor mínimo se observó en el ensayo 4, cuyos embriones no fueron viables ya que éstos no llegaron a estado de larva veliger fase $\mathrm{D}$; sin embargo, en el resto de los ensayos la fecundación de los ovocitos fue superior al $84 \%$, alcanzando todos al estado de larva veliger fase $\mathrm{D}$ a las $48 \mathrm{~h}$ post-fecundación, con supervivencias sobre el $88 \%$.

El estímulo de desove con serotonina no afectó la competencia/fertilidad de los gametos liberados. Gibbons \& Castagna (1984) mostraron que el suministro de serotonina en ostras, ostiones y almejas genera gametos competentes, fecundables, y que se transforman en larvas y consecutivamente son capaces de metamorfosear, lo que también fue observado en Haliotis asinina (Wang et al. 2010), Ilyanasa obsolete (Levantine \& Bonar 1986) y Pinctada maxima (Zhao et al. 2003) donde la serotonina fue el mejor inductor para supervivencia larval, asentamiento y metamorfosis. Esto también se observó en Tridacna gigas (Crawford et al. 1986), Hippoper porcelanus (Alcazar 1987) y los pectínidos del Caribe Argopecten nucleus y Nodipecten nodosus (Velasco et al. 2007). Hamida et al. (2004) demostraron también la competencia de ovocitos para ser fertilizados en Ruditapes decussatus, después de inducir la ruptura de la vesícula germinal incubándolos con serotonina. La no viabilidad de los embriones del ensayo 4 de este trabajo, puede ser atribuida a 2 factores: 1 ) la temperatura utilizada en el desove; E. macha es una especie muy sensible a temperaturas sobre $\operatorname{los} 17^{\circ} \mathrm{C}$ (Lépez et al. 2008), especialmente en el cultivo larval. Se ha reportado en $E$. arcuatus (Martínez \& DaCosta 2008) que temperaturas sobre $14^{\circ} \mathrm{C}$ afecta la supervivencia larval. En este estudio, el ensayo 4 se realizó a $18,4^{\circ} \mathrm{C}$, la más alta de todos los ensayos: 2) una posible poliespermia, pues se agregó la misma cantidad de solución de espermatozoides $(15 \mathrm{ml})$ empleada en los 5 ensayos, siendo que la liberación de ovocitos, en el ensayo 4, fue aproximadamente la mitad de los obtenidos en los otros ensayos. Avendaño et al. (2001) señalan que la poliespermia produce una alta mortalidad en las primeras $48 \mathrm{~h}$ de vida en $A$. purpuratus por un daño estructural a los ovocitos maduros de apariencia normal, lo que fue reportado también por Toro et al. (2010) en la misma especie.

El desove inducido con serotonina tiene la ventaja que es de fácil aplicación, no requiere ciclos de alternancia de temperatura, puede ser usado en forma independiente sin involucrar la mezcla de otros factores (por ejemplo, temperatura y alimento), es rápido y permite sincronizar la inducción a desove en los ejemplares. Esto facilita el manejo de los reproductores para obtener desoves individuales, necesarios en estudios genéticos (manipulación de stock) y de toxicidad. La serotonina puede ser fácilmente usada para inducir desove en especies que son resistentes a los estímulos tradicionales, como acontece en la mayoría de los bivalvos enterradores, en los cuales el estrés por temperatura no siempre asegura una respuesta positiva. Es por esto que los resultados de la presente investigación podrían ser empleados como una herramienta útil en los centros de producción de semillas de E. macha, ya que es efectiva y no altera la competencia de los gametos. En conclusión, los resultados sugieren que en reproductores maduros de Ensis macha, 5-HT tiene un efecto positivo en la liberación de espermatozoides y ovocitos competentes, con un patrón en el comportamiento de respuesta, siendo los machos los primeros en responder.

\section{Agradecimientos}

Los autores agradecen al BM Claudio Espinoza del Centro Referencia de estudios Ambientales de la Universidad Católica de la Santísima Concepción, por su aporte en la información de viabilidad de ovocitos fecundados, al BQ Víctor González del Dpto. de Botánica de la Universidad de Concepción, por su colaboración en la preparación de la solución de serotonina y a las Prof. Maritza Mora y Hanna Welna por revisar la traducción. Este estudio fue financiado por Celulosa Arauco y Constitución. 


\section{LITERATURA CITADA}

Alcazar S. 1987. Serotonin-induced spawning and larval rearing of the China clam, Hippopus porcellanus Rosewater (Bivalvia: Tridacnidae). Aquaculture 66(3-4): 359-368.

Aracena O, M Lépez, J Sánchez, A Carmona, L Medina \& A Saavedra. 2003. On two new macroscopic indexes to evaluate the reproductive cycle of Ensis macha (Molina, 1782). Journal of Shellfish Research 22(3): 675-680.

Avellanal M, E Jaramillo, E Clasing, P Quijon \& $\mathbf{H}$ Contreras. 2002. Reproductive cycle of the bivalves Ensis macha (Molina, 1782) (Solenidae), Tagelus dombeii (Lamarck, 1818) (Solecurtidae), and Mulinia edulis (King, 1831) (Mactridae) in southern Chile. Veliger 45(1): 33-44.

Avendaño M, M Le Pennec \& M Cantillánez. 2001. Anormalidades en larvas de Argopecten purpuratus (Lamarck, 1819) (Mollusca: Pectinidae), uno de los problemas en la producción artificial de semilla. Estudios Oceanológicos 20: 33-42.

Barón P, L Real, N Ciocco \& M Re. 2004. Morphometry, growth and reproduction of an Atlantic population of the razor clam Ensis macha (Molina, 1782). Scientia Marina 68: 211-217.

Bayne B. 1976. Aspects of reproduction in bivalve mollusks. In: Wiley M (ed). Estuarine Processes 1:432-448. Academic Press, London.

Belda C \& A Del Norte. 1988. Notes on the induced spawning and larval rearing of the Asian moon scallop, Amusium pleuronectes (Linné), in the laboratory. Aquaculture 72(12): $173-179$.

Braley R. 1985. Serotonin-induced spawning in giant clams (Bivalvia: Tridacnidae). Aquaculture 47: 321-325.

Campos E, F Bronfman, A Rivera, N Inestrosa \& G Martinez. 1992. Proceso reproductivo en invertebrados marinos: Neurotransmisores y AMP cíclico. Revista de Biología Marina 27: 225-234.

Crawford C, W Nash \& J Lucas. 1986. Spawning induction, and larval and juvenile rearing of the giant clam, Tridacna gigas. Aquaculture 58(3-4): 281-295.

Croll R, C Too, A Pani \& J Nason. 1995. Distribution of serotonin in the sea scallop Placopecten magellanicus. Invertebrate Reproduction \& Development 28:125-135.

Cubero E, C Ciocan, E Hill, M Osada, M Kishida, N Itoh, R Kondo, C Minier \& J Rotchell. 2010. Estrogens disrupt serotonin receptor and cyclooxygenase mRNA expression in the gonads of mussels (Mytilus edulis). Aquatic Toxicology 98(2): 178-187.

Darriba S \& C López. 2011. Anatomy. In: Guerra A, C Lodeiros, M Baptista \& FCosta (eds). Razor clams: biology, aquaculture and fisheries, pp. 45-65. Conselleria do Mar, Xunta de Galicia, Santiago de Compostela.

Darriba S, F San Juan \& A Guerra. 2004. Reproductive cycle of the razor clam Ensis arcuatus (Jeffreys, 1865) in northwest Spain and its relation to environmental conditions. Journal of Experimental Marine Biology and Ecology 311: 101-115.
Deguchi R \& K Osanai. 1995. Serotonin-induced meiosis reinitiation from the first prophase and from the first metaphase in oocytes of the marine bivalve Hiatella flaccida: respective changes in intracellular $\mathrm{Ca}^{2+}$ and $\mathrm{pH}$. Developmental Biology 171: 483-496.

Ferran P. 1993. Biología de moluscos bivalvos. En: Castelló F (ed). Acuicultura marina: fundamentos biológicos y tecnología de la producción, pp. 93-142. Universitat de Barcelona, Barcelona.

Flyachinskaya L. 2000. Localization of serotonin and FMRFamide in the bivalve mollusc Mytilus edulis at early stages of its development. Journal of Evolutionary Biochemistry and Physiology 36: 66-70.

Garnerot F, J Pellerin, C Blaise \& M Mathieu. 2006. Immunohistochemical localization of serotonin (5hydroxytryptamine) in the gonad and digestive gland of Mya arenaria (Mollusca: Bivalvia). General and Comparative Endocrinology 149(3): 278-284.

Gibbons MC \& M Castagna. 1984. Serotonin as an inducer of spawning in six bivalve species. Aquaculture 40: 189191.

Gobet I, Y Durocher, C Leclerc, M Moreau \& P Guerrier. 1994. Reception and transduction of the serotonin signal responsible for meiosisreinitiation in oocytes of the Japanese clam Ruditapes philippinarum. Developmental Biology 164: 540-549.

González M, O Parra \& A Cifuentes. 1995. Técnicas de cultivo de microalgas en laboratorio. En: Alveal K, ME Ferrario, EC Oliveira \& E Sar (eds). Manual de métodos ficológicos, pp. 219-250. Universidad de Concepción, Concepción.

Guerra A \& A Villagarcía. 1993. Cultivo intensivo de moluscos. En: Castelló F (ed). Acuicultura marina: fundamentos biológicos y tecnología de la producción, pp. 441-451. Universitat de Barcelona, Barcelona.

Hamida L, M Medhioub, J Cochard \& M Le Pennec. 2004. Evaluation of the effects of serotonin (5-HT) on oocyte competence in Ruditapes decussatus (Bivalvia, Veneridae). Aquaculture 239(1-4): 413-420.

Helm M, N Bourne \& A Lovatelli. 2006. Cultivo de bivalvos en criadero, un manual práctico. FAO Documento Técnico de Pesca 471: 1-184.

Kadam A \& S Koide. 1990. Stimulation of Spisula spermmotility by 5-hydroxytryptamine analogs. Invertebrate, Reproduction and Development 17: 33-37.

Leclerc C, P Guerrier \& M Moreau. 2000. Role of dihydropyridine-sensitive calcium channels in meiosis and fertilization in the bivalve mollusk Ruditapes philippinarum and Crassostreas gigas. Biology of the Cell 92: 285-299.

Lépez MI, F Vejar \& D Arriagada. 2011. Razor clam (Ensis macha) culture in Chile. In: Guerra A, C Lodeiros, M Baptista \& F Costa (eds). Razor clams: biology, aquaculture and fisheries, pp. 219-226. Conselleria do Mar, Xunta de Galicia. 
Levantine P \& D Bonar. 1986. Metamorphosis of Ilyanassa obsoleta: natural and artificial inducers. American Zoologist 26: $14 \mathrm{~A}$.

MartínezD \& F Da Costa. 2008. Cultivo de navaja, longueirón y longueirón vello. En: Guerra A\& C Lodeiros (eds). Navajas y longueirones: Biología, pesquerías y cultivo, pp. 187211. Conselleria do Mar, Xunta de Galicia.

Martínez G. 2008. Control de la reproducción y producción de semillas de bivalvos en sistemas controlados. En: Lovatelli A, A Farias \& I Uriarte (eds). Estado actual del cultivo y manejo de moluscos bivalvos y su proyección futura: factores que afectan su sustentabilidad en América Latina. Taller Técnico Regional de la FAO. 20-24 de agosto de 2007, Puerto Montt, Chile. FAO Actas de Pesca y Acuicultura 12: 267-275.

Martínez G, AZ Olivares \& L Mettifogo. 2000b. In vitro effects of monoamines and prostaglandins on meiosis reinitiation and oocyte release in Argopecten purpuratus Lamarck. Invertebrate, Reproduction and Development 38: 61-69.

Matsutani T \& T Nomura. 1982. Induction of spawning by serotonin in the scallop, Patinopecten yessoensis (Jay). Marine Biology Letters 3: 353-358.

Matsutani T \& T Nomura. 1987. In vitro effects of serotonin and prostaglandins on release of eggs from the ovary of the scallop, Patinopecten yessoensis. General and Comparative Endocrinology 67: 111-118.

Pani A \& R Croll. 1995. Distribution of catecholamines, indoleamines, and their precursors and metabolutes in the scallop, Placopecten magellanicus (Bivalvia, Pectinidae). Celular and Molecular Neurobiology 15: 371-386.

Paulet M, A Donval \& F Bekhadra. 1993. Monoamines and reproduction in Pecten maximus, a preliminary approach. Invertebrate Reproduction and Development 23: 89-94.

Quinn G \& M Keough. 2002. Experimental design and data analysis and biologist, 537 pp. Cambridge University Press, Cambridge.

Ram J, P Fong \& K Kyozuka. 1996. Serotoninergic mechanisms mediating spawning and oocyte maturation in the zebra mussel, Dressena polymorpha. Invertebrate Reproduction and Development 30: 29-37.
Ramírez J. 1993. Moluscos de Chile. 4: Bivalvia. (Tomos 1 y 2), 286 pp. Jaime Ramírez B, Santiago de Chile.

Sánchez M, E Estrada, M Bélanger, M Morissette \& T Di Paolo. 2011. Estradiol modulation of cortical, striatal and raphe nucleus 5-HT 1A and 5-HT 2A receptors of female hemiparkinsonian monkeys after long-term ovariectomy. Neuropharmacology 60(4): 642-652.

SERNAPESCA. 2011. Chile, desembarque total año 2011 por especie y por mes (en toneladas). Servicio Nacional de Pesca, Valparaíso. <http://www.sernapesca.cl/index.php?option $=$ com_remositoryItemid $=246 \&$ func $=$ startdown $\& i d=6323>$.

Toro J, M Montoya, V Martínez, D Gutiérrez \& A Vergara. 2010. Consecuencias de la autofecundación sobre la tasa de crecimiento y supervivencia de Argopecten purpuratus. Archivos de Medicina Veterinaria 42: 63-70.

Urban H. 1996. Population dynamics of the bivalves Venus antiqua, Tagelus dombeii, and Ensis macha from Chile at $36^{\circ}$ S. Journal of Shellfish Research 15: 719-727.

Velasco A, J Barros \& E Acosta. 2007. Spawning induction and early development of the Caribbean scallops Argopecten nucleus and Nodipecten nodosus. Aquaculture 266(1-4): 153-165.

Wang C. 2000. Roles of sex steroids on reproduction of the sea scallop. PhD thesis, Department of Physiology and Biophysics, Dalhousie University, Halifax, 274 pp.

Wang Ch \& R Croll. 2003. Effects of sex steroids on in vitro gamete release in the sea scallop, Placopecten magellanicus. Invertebrate Reproduction and Development 44: 89-100.

Wang X, Y Bai \& B Huang. 2010. Effect of chemical cues on larval survival, settlement and metamorphosis of abalone Haliotis asinine. Chinese Journal of Oceanology and Limnology 28(6): 1261-1265.

Zhao B, S Zhang \& P Qian. 2003. Larval settlement of the silver - or goldlip pearl oyster Pinctada maxima (Jameson) in response to natural biofilms and chemicals cues. Aquaculture 220: 883-901. 